\title{
Neuropsychiatric Complications of COVID-19
}

\author{
Zev M. Nakamura ${ }^{1,2}$ (D) $\cdot$ Rebekah P. Nash ${ }^{1} \cdot$ Sarah L. Laughon $^{1} \cdot$ Donald L. Rosenstein ${ }^{1,2,3}$ \\ Accepted: 23 February 2021 / Published online: 16 March 2021 \\ (C) The Author(s), under exclusive licence to Springer Science+Business Media, LLC, part of Springer Nature 2021
}

\begin{abstract}
Purpose of Review To describe the presentation, etiologies, and suggested management of post-acute COVID-19 neuropsychiatric symptoms.

Recent Findings Over 30\% of patients hospitalized with COVID-19 may exhibit cognitive impairment, depression, and anxiety that persist for months after discharge. These symptoms are even more common in patients who required intensive care for severe effects of the virus. In addition to the pandemic-related psychological stress, multiple biological mechanisms have been proposed to understand the neuropsychiatric symptoms observed with COVID-19. Given limited research regarding effective interventions, we recommend pharmacologic and behavioral strategies with established evidence in other medically-ill populations.

Summary Long-term, neuropsychiatric complications of COVID-19 are common and consequential. Because these are likely to co-occur with other medical problems, patients recovering from COVID-19 are best managed in clinics with highly coordinated care across disciplines and medical specialties. Future research is needed to inform appropriate interventions.
\end{abstract}

Keywords Neuropsychiatric $\cdot$ Neurocognitive $\cdot$ Mental health $\cdot$ COVID-19 $\cdot$ Post-COVID-19 $\cdot$ SARS-CoV-2

\section{Introduction}

The scope, magnitude, and speed of the COVID-19 pandemic have been staggering and continue to rapidly evolve. To date, more than 80 million people have been infected with the severe acute respiratory syndrome coronavirus-2 (SARS-CoV2) worldwide and at least 1.7 million have died [1]. Consequently, the full impact of this global infectious disease catastrophe is not likely to be appreciated for years to come. Early in the pandemic, public and scientific attentions were focused on the acute morbidity and mortality associated with COVID-19. However, several months into the pandemic,

This article is part of the Topical Collection on Psychiatry in Primary Care

Zev M. Nakamura

zev_nakamura@med.unc.edu

1 Department of Psychiatry, University of North Carolina at Chapel Hill, 101 Manning Drive, Campus Box \#7160, Chapel Hill, NC 27599, USA

2 Lineberger Comprehensive Cancer Center, University of North Carolina at Chapel Hill, Chapel Hill, NC, USA

3 Department of Medicine, University of North Carolina at Chapel Hill, Chapel Hill, NC, USA reports emerged describing persistent physical and neuropsychiatric sequelae in the aftermath of SARS-CoV-2 infection.

Whereas residual or persistent neuropsychiatric symptoms are not uncommon in critically ill survivors following admission to an intensive care unit (ICU) [2••], post-COVID-19 follow-up studies reveal that mild and even asymptomatic infection may lead to cognitive impairment, delirium, extreme fatigue, and clinically relevant mood symptoms [3, 4]. These descriptions mirror historical reports of post-pandemic neuropsychiatric complications such as encephalitis lethargica [5, 6], as well as descriptions of sequalae from other respiratory illness pandemics [7].

Recent evidence suggests that psychiatric illness is both a risk factor for and consequence of COVID-19. In a large electronic health record (EHR)-based cohort study of over 60,000 COVID-19 cases, a documented psychiatric diagnosis in the prior year was associated with a $65 \%$ increased risk of COVID-19 when compared with a matched cohort of patients with physical health issues without psychiatric diagnoses [8••]. Furthermore, over the 3 months following COVID-19 diagnosis, $18 \%$ of patients were diagnosed with a psychiatric diagnosis, with nearly $6 \%$ representing a new diagnosis (e.g., dementia, anxiety, and insomnia). Similar increases in incident psychiatric diagnoses among US adults with COVID19 were reported by Czeisler et al. [9॰], who also noted that specific populations were disproportionally affected (e.g., 
young adults, Hispanic and Black patients, essential workers, unpaid caregivers, and those with pre-existing psychiatric conditions).

This annotated review begins with an illustrative case and then summarizes the rapidly growing evidence base for persistent neuropsychiatric complications of COVID-19. Our aims are to describe post-acute COVID-19 neuropsychiatric complications, potential etiologies of these persistent central nervous system (CNS) symptoms, and provide recommendations for the evaluation and psychiatric management of recovering COVID-19 patients who present to primary care settings.

\section{Case Example}

A 62-year-old man with history of osteoarthritis, but otherwise no formal medical or psychiatric history, presented to the emergency department (ED) with a chief complaint of hip pain. In the ED, physical and mental status exam were relatively unremarkable. Specifically, the patient was afebrile, lung exam was normal, and his mental status was not grossly altered. Imaging and laboratory workup revealed an acute kidney injury (AKI, serum creatinine $1.7 \mathrm{mg} / \mathrm{dL}$ ), which prompted hospital admission. COVID-19 testing was performed as part of his admission lab work and returned positive. Family noted that one of the patient's sons had recently been exposed to COVID-19 at work and later tested positive. They also reported that the patient had been "confused" in the days preceding his presentation. Within hours of admission to the COVID-19 unit, the patient's creatinine normalized, but he quickly became belligerent, refusing interventions, and demanding to be discharged. The inpatient consulting psychiatrist diagnosed the patient with acute delirium and, based on history obtained from his family, a pre-existing mild neurocognitive disorder. Risperidone $0.5 \mathrm{mg}$ nightly was recommended for agitation, which gradually improved over his 5-day hospitalization. At time of discharge, the patient was connected with follow-up in a post-COVID clinic for ongoing management of his lingering neuropsychiatric symptoms, potentially precipitated by SARS-CoV-2 infection.

\section{Etiologies of Neuropsychiatric Symptoms}

SARS-CoV-2 is a positive sensed, single-stranded, enveloped RNA virus with a crown-like morphology [10]. It is a human coronavirus $(\mathrm{HCoV})$ in the beta genera of the coronaviridae family, along with severe acute respiratory syndrome coronavirus-1 (SARS-CoV-1), Middle East respiratory syndrome related coronavirus (MERS-CoV), $\mathrm{HCoV}-\mathrm{OC} 43$, and HCoV-KHU1 [10]. During cell entry, SARS-CoV-2 binds angiotensin converting-enzyme 2 (ACE2) on the target cell surface to facilitate fusion of viral and host membranes [11, 12]. ACE2 exists on cellular membranes in the lungs, gastrointestinal (GI) track, myocardium, renal tubules, and bladder
[12]. Correspondingly, coronaviruses traditionally are considered pulmonary diseases, often with accompanying GI symptoms [11].

However, COVID-19 patients have demonstrated a high prevalence of neuropsychiatric symptoms [8・•]. Of note, both SARS-CoV-1 and MERS-CoV have demonstrated the ability to infect the CNS, especially the brainstem [13]. Our knowledge of SARS-CoV-1 and MERS-CoV have contributed to four proposed mechanisms of CNS involvement by SARSCoV-2.

First, neuropsychiatric symptoms of COVID-19 are most commonly due to a myriad of biological and environmental factors, including electrolyte abnormalities, liver inflammation, impaired renal function, impaired oxygenation [14], hyperinflammation [12], and isolation due to public health concerns, which lead to a multifactorial delirium. Older individuals are at greatest risk for delirium due to these multiple contributors, and may experience both acute and long-term neuropsychiatric effects following an episode of delirium. $[15,16]$.

Second, viral-induced immune reaction and autoimmunity (during or after acute infection) provide another route by which SARS-CoV-2 can impact CNS function. While the virus is rarely found in the cerebrospinal fluid (CSF), a viralinduced inflammatory response can lead to blood brain barrier (BBB) dysfunction, resulting in immune cell infiltration and CNS tissue damage [12]. Indeed, there have been reports of limbic encephalitis during SARS-CoV-2 infection [17], as well as brainstem involvement [18].

Third, SARS-CoV-2-induced coagulopathy has resulted in a wide variety of organ failure. Viral invasion of vascular endothelium leading to activated thrombotic and inflammatory cascades in the midst of a hypercoagulable state can lead to cerebrovascular events [12]. Stroke is the most common neurological finding on imaging of hospitalized patients for SARS-CoV-2 [19]. Stroke can even be a presenting symptom, though is more typically part of multi-organ involvement [20]. Furthermore, stroke itself is a risk factor for depression, and COVID-19 patients with strokes are at significantly increased risk for poor outcomes [21].

Finally, direct viral invasion of the CNS has been demonstrated, though this insult appears to be uncommon. A few reports have identified the virus in the CNS, but this is rare, even among patients who are severely symptomatic $[22,23]$. Due to the well documented and prevalent loss of taste and smell in infected patients, direct CNS invasion by SARSCoV-2 through olfactory axonal migration was proposed. However, subsequent work has shown that it is actually olfactory epithelial cells, which provide metabolic support for the olfactory sensory neurons, rather than the neurons themselves that are most likely involved $[24,25]$. Therefore, direct SARSCoV-2 invasion of the CNS more likely occurs at the blood brain barrier (BBB) via (1) transcellular migration (through 
host endothelial cells); (2) paracellular migration (through tight junctions); and (3) an immune system "trojan horse" cell passing through the BBB [12].

\section{Neurocognitive Disorders}

There are few data regarding the long-term cognitive consequences of COVID-19. One study of 279 patients hospitalized with COVID-19 found that $34 \%$ reported memory loss and $28 \%$ described impaired concentration approximately 3 months after discharge [26]. Similar findings have been observed following infection with other coronaviruses, in which $20 \%$ reported cognitive deficits months to years after initial infection [7]. In Taquet et al's large EHR study, new onset dementia following hospitalization for COVID-19 was 2-3 times more common than what was observed after hospitalization for other medical events $[8 \bullet \bullet]$.

In more severe cases of COVID-19, long-term cognitive deficits are likely the sequelae of delirium experienced during the acute phases of illness. Particularly in older patients, as in our illustrative case, delirium is one of the most common symptoms in COVID-19 patients presenting to the ED, and can be the only or primary symptom of SARS-CoV-2 infection [15]. Delirium occurs in at least $30 \%$ of patients hospitalized with COVID-19 $[27 \bullet, 28 \cdot]$ and is substantially more common in those requiring ICU admission [29••]. The implications of delirium on long-term outcomes in these patients can be gleaned from the existing ICU literature. For example, a seminal study in adults with respiratory failure or shock admitted to the medical or surgical ICU identified global cognitive impairment (of equivalent severity to mild Alzheimer's disease) in $24 \%$ at 1 year after discharge [2]. Of interest, delirium has also been described in COVID-19 patients who do not experience severe medical complications (also as represented in our case) [27•,28•], and there have been reports of "brain fog" among patients experiencing milder symptoms who were never hospitalized and presumably did not experience delirium $[3,4]$.

The potentially prolonged and disabling neurocognitive effects of COVID-19 are of great relevance to providers practicing in primary care and outpatient specialist settings where these patients will be managed following hospitalization. Patients experiencing adverse cognitive effects of COVID-19 would benefit from longitudinal cognitive assessment with brief screening instruments (e.g., Montreal Cognitive Assessment [30]) and selfreport measures (e.g., Patient-Reported Outcomes Measurement Information System Cognitive Function [31]). Comorbidities that affect cognition (e.g., depression, anxiety, fatigue, sleep disturbance) and reversible causes (e.g., nutritional deficiencies, endocrinopathies, metabolic derangements) should be screened for and corrected as indicated. Patients with more pronounced symptoms may benefit from formal neuropsychological evaluation, which may in turn clarify the potential utility of cognitive rehabilitation or pharmacotherapies. To that end, there are no evidenced-based medications for neurocognitive problems due to COVID-19 (or similar conditions), but stimulants or medications used for the treatment of neurodegenerative disorders (e.g., acetylcholinesterase inhibitors, memantine) may be appropriate depending on patient comorbidities. Suggested management of neuropsychiatric symptoms following SARS-CoV-2 infection is presented in Table 1.

\section{Mood and Anxiety Disorders}

During and following COVID-19 infection, patients are at increased risk for depression and anxiety [32॰]. At approximately 1 month following infection, $31-38 \%$ of patients report depressive symptoms [33, 34], 22-42\% report anxiety symptoms [33, 34], and 20\% report obsessive-compulsive symptoms [33]. Rates vary depending on the population studied, methods used to evaluate symptoms, and how long after infection symptoms are assessed [32•]. Most studies are crosssectional and the majority are from China [32•]. One prospective study of patients in New York City described symptom severity during hospitalization and 14-17 days later. During hospitalization, $36 \%$ of participants reported anxiety and $29 \%$ reported depression; 14-17 days later, the prevalence of

Table 1 Common principles for management of neuropsychiatric complications of COVID-19 in the primary care setting

-Consider SARS-CoV-2 infection, in addition to pandemic-related stress, as a possible causative factor for new or worsening neuropsychiatric symptoms

-Recognize that patients with pre-existing psychiatric illness are more likely to become infected with SARS-CoV-2, experience neuropsychiatric consequences of COVID-19, and suffer worse medical outcomes

-Symptoms should be monitored longitudinally, at regular intervals, using validated rating scales and questionnaires to screen for depression, anxiety, post-traumatic stress, substance use, suicidality, and cognitive concerns

-Evidence-based behavioral and pharmacological therapies used to address symptoms in other medically ill populations can be used in the setting of an active or resolving SARS-CoV-2 infection, but providers should be aware of potential drug-drug interactions, particularly in light of COVID-19's pro-inflammatory, pro-thrombotic, and arrhythmogenic effects

-Given the multiple organ systems affected by COVID-19, we recommend highly coordinated care alongside other specialists (e.g., cardiology, nephrology, infectious disease, pulmonology, neurology, rehabilitation medicine)

-Telehealth represents an opportunity to expand access to mental health care while mitigating the spread of the virus; however, providers should be mindful of the limited research regarding the efficacy of telepsychiatry for certain populations (e.g., psychotic disorders) and develop plans for necessary face-to-face care (e.g., administration of long-acting injectable medications, methadone treatment programs) 
anxiety and depression had decreased (9\% and 20\%, respectively), but acute stress symptoms emerged in $25 \%$ [35•].

While depressive and anxiety symptoms are more common than formal mood or anxiety diagnoses in COVID-19 survivors, a not insignificant proportion of patients meet diagnostic criteria for a psychiatric disorder. In the study by Taquet et al., a new or recurrent anxiety disorder was documented in $12.8 \%$ and depressive disorder in $9.9 \%$. Severe COVID-19 disease was associated with higher rates of these diagnoses, but all patients with COVID-19 had higher rates of psychiatric diagnoses when compared to controls [8••]. Depressive and/or anxiety symptoms following COVID-19 are more likely in females [33, 36, 37] and those with infected family members [34, 36, 37], post-infection physical discomfort [34], severe infection [36], elevated inflammatory markers [33, 38], and prior psychiatric diagnoses [33].

Suicidal ideation is also elevated following COVID-19 diagnosis. In one study, $3.5 \%$ of respondents reported suicidality at 1 month following infection [33]. There have been numerous case reports of COVID-19 patients attempting to kill themselves during or prior to hospitalization $[13,39$, 40]. Nonetheless, it remains prudent to withhold final judgment on the relationship between COVID-19 and suicide until definitive epidemiological studies can be completed [41].

There have been few interventional studies targeting anxiety and depression in COVID-19 patients. Progressive muscle relaxation training $[42,43]$ and an internet-based integrated intervention (including self-help with relaxation, mindfulness, self-compassion skills) [44] were both associated with lower depression and anxiety scale scores in patients admitted to Chinese hospitals. Due to the reported relationship between psychological resilience and lower anxiety and depression [45], psychotherapeutic approaches that enhance resilience would appear beneficial. To our knowledge, there are no well-defined pharmacologic guidelines for management of depression and anxiety following SARS-CoV-2 infection [46•]. However, the general approach to psychiatric symptom management in the medically ill can also be applied to this population. First, the goal is to avoid harm by considering the various organs that might have been compromised by the virus, and then either withhold medications, or adjust doses, accordingly. In many outpatients, hydroxyzine is a good first option for as needed anxiety management. If renal function is preserved, gabapentin, on a scheduled or as needed basis, can be efficacious for anxiety [46 ${ }^{\bullet}$. Selective serotonin reuptake inhibitors (SSRIs) are generally well-tolerated options for depression and anxiety, though paroxetine is not recommended due to its short half-life, increased risk of drug-drug interactions, and withdrawal symptoms.

\section{Post-Traumatic Stress Disorder}

Post-traumatic stress disorder (PTSD) was one of the most common psychiatric disorders diagnosed among survivors of SARS and MERS, with a prevalence of nearly $40 \%$ at a 6 -month postdischarge [47•]. Not surprisingly, the prevalence of PTSD among COVID-19 survivors is anticipated to be at least as high as with previous coronavirus outbreaks [7, 48, 49].

To date, the prevalence of PTSD among COVID-19 patients appears to range from 20 to $30 \%$, while the prevalence of less strictly defined post-traumatic stress symptoms (PTSS) varies widely [50-53]. For example, in a study of more than 700 clinically stable, hospitalized COVID-19 patients in China, 96.2\% reported significant PTSS associated with COVID-19 [51], where PTSS were observed in only $31 \%$ in a British cohort evaluated 4-8 weeks after discharge from a large university hospital [50]. By comparison, in a study of 64 Korean COVID-19 patients interviewed approximately 2.5 months following hospital discharge, 20.3\% met criteria for PTSD [52]. And similarly, in a cohort of 185 Italian patients who had been hospitalized with COVID-19, 22.2\% met criteria for PTSD at a 2-week post-discharge follow-up visit [53].

The most common risk factors identified to date for PTSD/PTSS following SARS-CoV-2 infection are younger age [50, 53], female gender [53], need for ICU-level care [50], and having a past psychiatric history [53]. Interestingly, in a study comparing patients with COVID-19 who were discharged from the ED to those who required inpatient hospitalization, being hospitalized was found to protect against PTSD [53]. Among patients admitted to the ICU, obesity increased risk for PTSD, but this association was not observed among patients admitted to general medical wards [50].

Of interest, many risk factors for COVID-19 are also risk factors for PTSD. Specifically, high rates of obesity, diabetes, metabolic syndrome, cardiovascular disease, and autoimmune disease are observed in patients with PTSD [54-56]. COVID19 patients also have disproportionately high rates of medical comorbidities, including obesity, diabetes, chronic pulmonary and cardiac disease, as well as immune dysfunction [57-59]. Delirium and ICU-level care, both common complications of COVID-19 [16, 26, 60], are also risk factors for PTSD/PTSS, with approximately $20 \%$ of critical care survivors experiencing PTSS at 12-months after discharge [61].

To our knowledge, pharmacologic treatment specific to PTSD/PTSS related to COVID-19 has not been studied. However, as discussed in our recommendations for management of anxiety and depression, basic principles of treatment for PTSD in the medically ill can be applied, considering potential drug-drug interactions and possible organ dysfunction when selecting medications and doses. While evidence supports use of the serotonin-norepinephrine reuptake inhibitor (SNRI) venlafaxine and SSRIs for PTSD in medically ill patients, potential risks should be carefully considered on a case-to-case basis [62]. Again, paroxetine is not recommended in medically ill patients due to its short half-life, anticholinergic side effect profile, and higher risk for drug-drug interactions. Several studies have shown a reduction in nightmare 
frequency and intensity, as well as improvement in other PTSD symptoms, in patients prescribed the alpha- 1 receptor blocker, prazosin [63]. There is some evidence that psychoeducational services provided online to COVID-19 survivors with PTSS have been helpful, though restricted Internet access and poor health status in many affected patients make in-person psychological interventions preferred when possible [51, 53]. Exposure-based cognitive behavioral therapy (CBT) has the highest level of evidence in those with PTSD, while supportive counseling, resilience training and psychological first aid have some evidence in treating PTSD [64]. Of note, psychological debriefing is not beneficial for PTSD, may cause harm, and should be avoided [64].

\section{Psychotic Disorders}

Beginning with the Spanish Influenza pandemic in 1918, higher rates of psychosis have been observed during many pandemics or epidemics [65]. Early in the COVID-19 pandemic, an observational study from China reported a $25 \%$ increased incidence of psychotic disorders [66]. This relationship has been largely attributed to the substantial psychosocial stress of the pandemic, but, as described previously, more direct mechanisms have also been suspected. Of interest, $0.9-4 \%$ of people exposed to viral infections develop psychosis [67], which is much higher than the incidence in the general population of 15.2 in 100,000 persons [68]. The ongoing CoroNerve surveillance study identified new-onset psychosis in 10 of the first 153 patients with acute COVID-19-related neuropsychiatric complications [28•]. There have been several cases from multiple countries detailing first-episode psychotic symptoms in COVID-19 patients [69-72]. There are insufficient data to clarify a typical presentation of COVID-19 psychosis, but notable disorganization and confusional features have been described $[71,72]$. Compared to patients who develop psychosis precipitated by pandemic-related stress, these patients may be less likely to endorse paranoia or delusional content about COVID-19 [69]. They may also be less likely to have a family history of psychosis and more likely to present at an atypical age with subacute onset and relatively fast recovery following treatment with low-dose antipsychotics [71].

COVID-19 treatment may also precipitate psychosis. Specifically, chloroquine and hydroxychloroquine, which were previous mainstays of COVID-19 care, can cause hallucinations and other psychotic symptoms [73]. This risk is exacerbated in patients receiving combination therapy with lopinavir/ritonavir due to inhibition of CYP3A4 [73]. The administration of high-dose corticosteroids, which remains one of few effective treatments for severe COVID-19 infection, can cause psychotic symptoms, which has also been described specifically in the setting of treatment of viral illnesses $[74,75]$.
It is important to consider COVID-19 as a cause of new-onset psychotic symptoms [69]. Patients with COVID-19-related psychosis may exhibit elevations in CRP, ferritin, LDH, and DDimer, as well as either elevated or depressed WBC or platelets levels $[71,76,77]$. Low-dose antipsychotic medications may be helpful $[67,69,71,72]$, and patients hospitalized for severe COVID-19 may present to primary care settings on antipsychotics that were started during the acute period [71]. It is important to be mindful that antipsychotics increase risk of QT prolongation and Torsades de Pointes, particularly when used in conjunction with other QT prolonging medications (e.g., azithromycin). Additionally, COVID-19 infection itself is proarrhythmogenic [78]. Similarly, cerebrovascular complications are exceedingly common in severe COVID-19 [28•, 79], and exposure to antipsychotics may compound this risk [80]. While telehealth is an invaluable way of delivering care for patients during the pandemic, patients with psychotic disorders have lower utilization of mobile phones and technology compared to the general population [81] and there is a dearth of research regarding telepsychiatry interventions for patients with psychotic disorders [82].

\section{Substance Use Disorders}

When COVID-19 was declared a global pandemic, the USA was already in the midst of another public health crisis - the opioid epidemic. By June of 2020, the CDC reported that approximately one out of every 10 Americans had either started using illicit substances or increased the quantity and/ or frequency of substance use because of the COVID-19 pandemic [9]. Not surprisingly, it is anticipated there will be more drug overdose deaths in 2020 than in any year prior.

Screening for substance use disorders (SUDs) will be an important part of follow-up care for COVID-19 survivors. Patients with SUDs are more susceptible to contracting COVID-19 due to the social and public restrictions implemented during the pandemic as compared to those without SUDs [83-89]. Across all patients with COVID-19, those with comorbid SUDs are more likely to require hospitalization ( $40.1 \%$ vs $30.1 \%)$ or die $(9.6 \%$ vs $6.6 \%$ ) [89••]. These adverse outcomes are disproportionately higher in African American patients [89••].

Both COVID-19 and illicit substances, especially opioids, can profoundly impair pulmonary function [90-92]. Patients with SUDs are more likely to have risk factors for severe COVID-19 disease (e.g., obesity, type-2 diabetes, cancer, cardiovascular diseases, and chronic liver, kidney, and lung disease) compared to those without SUDs [89••, 90, 91] . Additional biological mechanisms for worse outcomes in this vulnerable subset of COVID-19 patients include suppressed immune function due to complex opioid immune modulation, and drug interactions between the medications used to treat opioid use disorder and COVID-19 [92]. 
COVID-19 survivors need to be screened for SUDs and, when present, providers should encourage harm-reduction strategies if abstinence is not possible. Strategies to minimize harm include having a surplus of clean syringes and other supplies, not sharing syringes, pipes or other paraphernalia, having naloxone readily available, and having a back-up plan for how to get medical attention if emergency services are needed given possible increased response time due to the overwhelming volume on health care facilities [83, 87]. Medication-assisted treatment (MAT) with buprenorphine or methadone is first-line treatment for patients with opioid use disorder and measures should be taken to ensure patients have access to MAT [93, 94•]. Increased MAT waivers and alleviation of prescribing restrictions have facilitated access during the pandemic [86].

\section{Post-COVID Clinical Infrastructure}

Interdisciplinary post-COVID-19 clinics are currently being established in multiple medical centers $[27 \bullet, 95]$. At the University of North Carolina at Chapel Hill, a "COVID Recovery Clinic" was recently created. Since COVID-19 affects multiple organ systems and will require follow-up care across multiple specialties, this clinic aims to provide care in a systematic and carefully coordinated fashion. The purpose of the clinic is to support adult COVID-19 survivors who experience ongoing medical complications, residual symptoms, and/or loss of functional independence. The clinic integrates providers with complementary expertise to improve patient care and provide educational outreach to healthcare professionals and the community. The lead specialty for the clinic is Physical Medicine and Rehabilitation. The core clinic group is comprised of clinicians from internal medicine, psychiatry, neuropsychology, physical therapy, occupational health, and speech therapy. There is also a collaborative group that includes cardiology, nephrology, infectious disease, pulmonary, geriatrics, neurology, nutrition, and other specialists. Patients are screened over the telephone with a battery of validated instruments and, as indicated, evaluated in-person in clinic for further assessment and management. In addition to the goal of providing exceptional clinical care for patients affected by sequelae of COVID-19, the clinic has been designed to facilitate unique teaching and research opportunities.

\section{Conclusion}

Scientific understanding of the impact of COVID-19 continues to evolve rapidly. There are emerging data concerning for a wide range of neuropsychiatric sequelae following SARS-CoV-2 infection. Due to the complexity of COVID19 and its treatment, affected patients may require longitudinal follow-up most appropriately delivered by multi-disciplinary teams. To accelerate medical knowledge regarding the longterm effects of COVID-19, it would be extremely fruitful for these post-COVID clinics to pool data into shared databases. Clinical trials are also urgently needed to clarify optimal treatment strategies for neuropsychiatric and other potentially long-term impacts of COVID-19.

\section{Compliance with Ethical Standards}

Conflict of Interest The authors declare that they have no conflicts of interest.

Human and Animal Rights and Informed Consent This article does not contain any studies with human or animal subjects performed by any of the authors.

\section{References}

Papers of particular interest, published recently, have been highlighted as:

- Of importance

•. Of major importance

1. Johns Hopkins University \& Medicine. COVID-19 Dashboard by the Center for Systems Science and Engineering (CSSE) at Johns Hopkins University (JHU) [Internet]. 2020. Available from: https:// coronavirus.jhu.edu/map.html

2.• Pandharipande PP, Girard TD, Jackson JC, Morandi A, Thompson JL, Pun BT, et al. Long-term cognitive impairment after critical illness. N Engl J Med Mass Medical Soc. 2013;369:1306-16. Cognitive function follow-up study of over 800 patients cared for in medical or surgical intensive care units. Nearly $75 \%$ developed delirium during the hospital stay, which was associated with substantial cognitive impairment (comparable to mild Alzheimer's Disease) in one quarter of patients evaluated 12 months after discharge.

3. Rubin R. As their numbers grow, COVID-19 "long haulers" stump experts. JAMA. 2020;324:1381-3.

4. Woo MS, Malsy J, Pöttgen J, Zai SS, Ufer F, Hadjilaou A, et al Frequent neurocognitive deficits after recovery from mild COVID19. Brain Commun. 2020;2:fcaa205.

5. Hoffman LA, Vilensky JA. Encephalitis lethargica: 100 years after the epidemic. Brain. 2017;140:2246-51.

6. Triarhou LC. The signalling contributions of Constantin von Economo to basic, clinical and evolutionary neuroscience. Brain Res Bull Elsevier. 2006;69:223-43.

7. Rogers JP, Chesney E, Oliver D, Pollak TA, McGuire P, Fusar-Poli $\mathrm{P}$, et al. Psychiatric and neuropsychiatric presentations associated with severe coronavirus infections: a systematic review and metaanalysis with comparison to the COVID-19 pandemic. Lancet Psychiatry. 2020;7:611-27.

8.• Taquet M, Luciano S, Geddes JR, Harrison PJ. Bidirectional associations between COVID-19 and psychiatric disorder: a study of 62,354 COVID-19 cases. Lancet Psychiatry. 2020;S2215-0366: 30462-4. A retrospective cohort study, using the large TriNetX Analytics Network, examined electronic health records from 54 US institutions to compare presence of psychiatric diagnoses following COVID-19 diagnosis relative to other 
health events over the first half of 2020. COVID-19 diagnosis was associated with increased likelihood of both new and recurrent psychiatric diagnoses relative to all other health events.

9. Czeisler MÉ, Lane RI, Petrosky E, Wiley JF, Christensen A, Njai R, et al Mental health, substance use, and suicidal ideation during the COVID-19 pandemic - United States, June 24-30, 2020. MMWR Morb Mortal Wkly Rep [Internet]. Centers for Disease Control and Prevention; 2020;69:1049-57. Available from: https://pubmed. ncbi.nlm.nih.gov/32790653. A report of increased adverse mental health conditions, substance abuse, and suicidal ideation in the United States since the early weeks of the pandemic in 2020.

10. Ye ZW, Yuan S, Yuen KS, Fung SY, Chan CP, Jin DY. Zoonotic origins of human coronaviruses. Int J Biol Sci. 2020;16:1686-97.

11. Jasti M, Nalleballe K, Dandu V, Onteddu S. A review of pathophysiology and neuropsychiatric manifestations of COVID-19. J Neurol Springer. 2020;Jun;3(1):6.

12. Achar A, Ghosh C. COVID-19-associated neurological disorders: the potential route of CNS invasion and blood-brain relevance. Cells. 2020;9:2360.

13. Liu Y, Cao L, Li X, Jia Y, Xia H. Awareness of mental health problems in patients with coronavirus disease 19 (COVID-19): a lesson from an adult man attempting suicide. Asian J Psychiatr Elsevier. 2020;51:102106.

14. Oussalah A, Gleye S, Urmes IC, Laugel E, Barbé F, Orlowski S, et al. The spectrum of biochemical alterations associated with organ dysfunction and inflammatory status and their association with disease outcomes in severe COVID-19: a longitudinal cohort and time-series design study. EClinicalMedicine. Elsevier. 2020;27: 100554.

15. Kennedy M, Helfand BKI, Gou RY, Gartaganis SL, Webb M, Moccia JM, et al. Delirium in older patients with COVID-19 presenting to the emergency department. JAMA Netw Open. 2020, 2020;(3):e2029540-0. https://doi.org/10.1001/jamanetworkopen. 2020.29540 .

16. Helms J, Kremer S, Merdji H, Schenck M, Severac F, Clere-Jehl R, et al. Delirium and encephalopathy in severe COVID-19: a cohort analysis of ICU patients. Crit Care BioMed Central. 2020;24:1-11.

17. Zambreanu L, Lightbody S, Bhandari M, Hoskote C, Kandil H, Houlihan CF, et al. A case of limbic encephalitis associated with asymptomatic COVID-19 infection. J Neurol Neurosurg Psychiatry. 2020;91:1229-30.

18. Khoo A, McLoughlin B, Cheema S, Weil RS, Lambert C, Manji H, et al. Postinfectious brainstem encephalitis associated with SARSCoV-2. J Neurol Neurosurg Psychiatry. 2020;91:1013-4.

19. Jain R, Young M, Dogra S, Kennedy H, Nguyen V, Jones S, et al. COVID-19 related neuroimaging findings: a signal of thromboembolic complications and a strong prognostic marker of poor patient outcome. J Neurol Sci Elsevier. 2020;116923.

20. Diaz-Segarra N, Edmond A, Kunac A, Yonclas P. COVID-19 ischemic strokes as an emerging rehabilitation population: a case series. Am J Phys Med Rehabil. 2020;99:876-9.

21. Ghannam M, Alshaer Q, Al-Chalabi M, Zakarna L, Robertson J, Manousakis G. Neurological involvement of coronavirus disease 2019: a systematic review. J Neurol. 2020;267:3135-53.

22. Moriguchi T, Harii N, Goto J, Harada D, Sugawara H, Takamino J, et al. A first case of meningitis/encephalitis associated with SARSCoronavirus-2. Int J Infect Dis Elsevier. 2020;94:55-8.

23. Al Saiegh F, Ghosh R, Leibold A, Avery MB, Schmidt RF, Theofanis T, et al. Status of SARS-CoV-2 in cerebrospinal fluid of patients with COVID-19 and stroke. J Neurol Neurosurg Psychiatry. 2020;91:846-8.

24. Gupta K, Mohanty SK, Mittal A, Kalra S, Kumar S, Mishra T, et al The cellular basis of loss of smell in 2019-nCoV-infected individuals. Brief Bioinform. Oxford University Press; 2020;Aug 18: bbaa168.
25. Brann DH, Tsukahara T, Weinreb C, Lipovsek M, Van den Berge K, Gong B, et al Non-neuronal expression of SARS-CoV-2 entry genes in the olfaory system suggests mechanisms underlying COVID-19associated anosmia. Sci Adv. American Association for the Advancement of Science; 2020;eabc5801.

26. Garrigues E, Janvier P, Kherabi Y, Le Bot A, Hamon A, Gouze H, et al. Post-discharge persistent symptoms and health-related quality of life after hospitalization for COVID-19. J Inf Secur. 2020;81:e4-6.

27. Liotta EM, Batra A, Clark JR, Shlobin NA, Hoffman SC, Orban ZS, et al. Frequent neurologic manifestations and encephalopathyassociated morbidity in Covid-19 patients. Ann Clin Transl Neurol. 2020;7:2221-30. This report of neurologic outcomes in a series of over 500 consecutive COVID-19 patients in a Chicago-based hospital network found neurologic manifestations in approximately $80 \%$ of patients at some time during their disease course.

28. Varatharaj A, Thomas N, Ellul MA, NWS D, Pollak TA, Tenorio EL, et al. Neurological and neuropsychiatric complications of COVID-19 in 153 patients: a UK-wide surveillance study. Lancet Psychiatry. 2020;7:875-82. This nationwide surveillance study described the development of and initial findings from an online portal which received real-time case report notifications of neuropsychiatric symptoms from patients presenting across the United Kingdom. Among the first 153 patients with complete clinical data sets, $31 \%$ presented with "altered mental status", which included new-onset psychosis, dementia-like syndromes, and affective disorders.

29.• Helms J, Kremer S, Merdji H, Clere-Jehl R, Schenck M, Kummerlen C, et al. Neurologic features in severe SARS-CoV-2 infection. N Engl J Med. 2020;382:2268-70. This report of 58 consecutive patients admitted with severe SARS-CoV-2 infection is one of the first to describe the neuropsychiatric effects of COVID-19. Among many widely cited findings from this study is the presence of a dysexecutive syndrome in $33 \%$ of patients at the time of hospital discharge.

30. Nasreddine ZS, Phillips NA, Bédirian V, Charbonneau S, Whitehead V, Collin I, et al. The Montreal Cognitive Assessment, MoCA: a brief screening tool for mild cognitive impairment. J Am Geriatr Soc. 2005;53:695-9.

31. Cella D, Riley W, Stone A, Rothrock N, Reeve B, Yount S, et al. The Patient-Reported Outcomes Measurement Information System (PROMIS) developed and tested its first wave of adult self-reported health outcome item banks: 2005-2008. J Clin Epidemiol. 2010;63: 1179-94 Available from: http://www.sciencedirect.com/science/ article/pii/S0895435610001733.

32. Deng J, Zhou F, Hou W, Silver Z, Wong CY, Chang O, et al. The prevalence of depression, anxiety, and sleep disturbances in COVID-19 patients: a meta-analysis. Ann N Y Acad Sci. WileyBlackwell; 2020;Oct 2:https://doi.org/10.1111/nyas.14506. A meta-analysis performed in August 2020 found significantly elevated prevalence for depression $(45 \%)$, anxiety $(47 \%)$, and sleep disturbance $(34 \%)$ in patients with COVID-19; the literature reviewed was dominated by Chinese studies.

33. Mazza MG, De Lorenzo R, Conte C, Poletti S, Vai B, Bollettini I, et al. Anxiety and depression in COVID-19 survivors: role of inflammatory and clinical predictors. Brain Behav Immun Elsevier. 2020;89:594-600.

34. Cai X, Hu X, Ekumi IO, Wang J, An Y, Li Z, et al. Psychological distress and its correlates among COVID-19 survivors during early convalescence across age groups. Am J Geriatr Psychiatry Elsevier. 2020;28:1030-9.

35. Parker C, Shalev D, Hsu I, Shenoy A, Cheung S, Nash S, et al. Depression, anxiety, and acute stress disorder among patients hospitalized with coronavirus disease 2019: a prospective cohort study. Psychosomatics Elsevier. 2020;S0033:3182-30262-0 In one of the few prospective studies of psychiatric symptoms in 
COVID-19 survivors, the authors tracked symptom severity over 2 weeks, from hospitalization to post-acute care. Depression severity remained stable over this time, while anxiety severity improved; acute stress symptoms were prominent following post-acute care.

36. Ma Y-F, Li W, Deng H-B, Wang L, Wang Y, Wang P-H, et al. Prevalence of depression and its association with quality of life in clinically stable patients with COVID-19. J Affect Disord Elsevier. 2020;275:145-8.

37. Nie X-D, Wang Q, Wang M-N, Zhao S, Liu L, Zhu Y-L, et al Anxiety and depression and its correlates in patients with coronavirus disease 2019 in Wuhan. Int J Psychiatry Clin Pract. Taylor \& Francis; 2020;1-6.

38. Guo Q, Zheng Y, Shi J, Wang J, Li G, Li C, et al. Immediate psychological distress in quarantined patients with COVID-19 and its association with peripheral inflammation: a mixed-method study. Brain Behav Immun Elsevier. 2020;88:17-27.

39. Epstein D, Andrawis W, Lipsky AM, Ziad HA, Matan M. Anxiety and suicidality in a hospitalized patient with COVID-19 infection. Eur J case reports Intern Med. 2020;7:001651.

40. Gillett G, Jordan I. Severe psychiatric disturbance and attempted suicide in a patient with COVID-19 and no psychiatric history. BMJ Case Reports CP. BMJ Specialist Journals. 2020;13:e239191.

41. John A, Pirkis J, Gunnell D, Appleby L, Morrissey J. Trends in suicide during the covid-19 pandemic. Br Med J. 2020;371:m4352.

42. Xiao C-X, Lin Y-J, Lin R-Q, Liu A-N, Zhong G-Q, Lan C-F. Effects of progressive muscle relaxation training on negative emotions and sleep quality in COVID-19 patients: a clinical observational study. Medicine (Baltimore). 2020;99:e23185.

43. Liu K, Chen Y, Wu D, Lin R, Wang Z, Pan L. Effects of progressive muscle relaxation on anxiety and sleep quality in patients with COVID-19. Complement Ther Clin Pract Elsevier. 2020;39: 101132.

44. Wei N, Huang B, Lu S, Hu J, Zhou X, Hu C, et al. Efficacy of internet-based integrated intervention on depression and anxiety symptoms in patients with COVID-19. J Zhejiang Univ Sci B Springer. 2020;21:400-4.

45. Zhang J, Yang Z, Wang X, Li J, Dong L, Wang F, et al. The relationship between resilience, anxiety and depression among patients with mild symptoms of COVID-19 in China: a cross-sectional study. J Clin Nurs Wiley Online Library. 2020;29:4020-9.

46. Khawam E, Khouli H, Pozuelo L. Treating acute anxiety in patients with COVID-19. Cleve Clin J Med. 2020;May 14:doi:https://doi. org/10.3929/ccjm.87a.ccc016. This annotated review of proposed therapies, including both psychotropic medications and psychotherapeutics, for psychiatric symptoms in COVID19 patients found only a limited number of evidence-based studies for this population.

47. Ahmed H, Patel K, Greenwood DC, Halpin S, Lewthwaite P, Salawu A, et al. Long-term clinical outcomes in survivors of severe acute respiratory syndrome and Middle East respiratory syndrome coronavirus outbreaks after hospitalisation or ICU admission: a systematic review and meta-analysis. J Rehabil Med. Foundation for Rehabilitation Information; 2020;52:jrm00063. This systematic review of 28 studies identified physical and psychological impairments among SARS and MERS survivors to inform clinicians of what to anticipate in COVID-19 survivors. Impaired lung function, reduced exercise capacity, and high rates of PTSD, depression and anxiety were the most common complications up to 6 months post-infections.

48. Han RH, Schmidt MN, Waits WM, Bell AKC, Miller TL. Planning for mental health needs during COVID-19. Curr Psychiatry Rep. Springer. 2020;22:66

49. Krishnamoorthy Y, Nagarajan R, Saya GK, Menon V. Prevalence of psychological morbidities among general population, healthcare workers and COVID-19 patients amidst the COVID-19 pandemic: a systematic review and meta-analysis. Psychiatry Res. Elsevier. 2020;293:113382.

50. Halpin SJ, McIvor C, Whyatt G, Adams A, Harvey O, McLean L, et al Postdischarge symptoms and rehabilitation needs in survivors of COVID-19 infection: a cross-sectional evaluation. J Med Virol. 2020;Jul 30:doi:https://doi.org/10.1002/jmv.26368.

51. Bo HX, Li W, Yang Y, Wang Y, Zhang Q, Cheung T, et al Posttraumatic stress symptoms and attitude toward crisis mental health services among clinically stable patients with COVID-19 in China. Psychol Med. Cambridge University Press; 2020;Mar 27:doi:https://doi.org/10.1017/S0033291720000999.

52. Chang MC, Park D. Incidence of post-traumatic stress disorder after coronavirus disease. Healthcare (Basel). 2020;8:373.

53. Yang Y, Li W, Zhang Q, Zhang L, Cheung T, Xiang YT. Mental health services for older adults in China during the COVID-19 outbreak. Lancet Psychiatry. 2020;7:e19.

54. Michopoulos V, Vester A, Neigh G. Posttraumatic stress disorder: A metabolic disorder in disguise. Exp Neurol Academic Press Inc. 2016;284:220-9.

55. Rosenbaum S, Stubbs B, Ward PB, Steel Z, Lederman O, Vancampfort $\mathrm{D}$. The prevalence and risk of metabolic syndrome and its components among people with posttraumatic stress disorder: a systematic review and meta-analysis. Metabolism. 2015;64: 926-33.

56. Vilchinsky N, Ginzburg K, Fait K, Foa EB. Cardiac-diseaseinduced PTSD (CDI-PTSD): a systematic review. Clin Psychol Rev. 2017;55:92-106.

57. Docherty AB, Harrison EM, Green CA, Hardwick HE, Pius R, Norman L, et al. Features of 20133 UK patients in hospital with covid-19 using the ISARIC WHO clinical characterisation protocol: prospective observational cohort study. BMJ. 2020;369:m1985.

58. Flaherty GT, Hession P, Liew CH, Lim BCW, Leong TK, Lim V, et al. COVID-19 in adult patients with pre-existing chronic cardiac, respiratory and metabolic disease: a critical literature review with clinical recommendations. Trop Dis Travel Med Vaccines. 2020;6:16.

59. Jergović M, Bendelja K, Mlakar AS, Vojvoda V, Aberle N, Jovanovic $\mathrm{T}$, et al. Circulating levels of hormones, lipids, and immune mediators in posttraumatic stress disorder - a three-month follow-up study. Front Psychiatry. 2015;6:1-13.

60. Garcez FB, Aliberti MJR, Poco PCE, Hiratsuka M, Takahashi S, de F Coelho VA, et al. Delirium and adverse outcomes in hospitalized patients with COVID-19. J Am Geriatr Soc. 2020;68:2440-6.

61. Righy C, Rosa RG, Da Silva RTA, Kochhann R, Migliavaca CB, Robinson CC, et al. Prevalence of post-traumatic stress disorder symptoms in adult critical care survivors: a systematic review and meta-analysis. Crit Care Critical Care. 2019;23:1-13.

62. Ravindran LN, Stein MB. Pharmacotherapy of PTSD: premises, principles, and priorities. Brain Res. 2009;1293:24-39.

63. Raskind MA, Peskind ER, Chow B, Harris C, Davis-Karim A, Holmes HA, et al. Trial of prazosin for post-traumatic stress disorder in military veterans. N Engl J Med. 2018;378:507-17.

64. Freedman SA. Psychological interventions to prevent PTSD. Psychiatr Ann. 2019;49:314-9.

65. Kępińska AP, Iyegbe CO, Vernon AC, Yolken R, Murray RM, Pollak TA. Schizophrenia and influenza at the centenary of the 1918-1919 Spanish influenza pandemic: mechanisms of psychosis risk. Front Psychiatry. 2020;11:72.

66. Hu W, Su L, Qiao J, Zhu J, Zhou Y. COVID-19 outbreak increased risk of schizophrenia in aged adults. PsychChinaXiv. 2020;10.202003.00003.

67. Brown E, Gray R, Lo Monaco S, O’Donoghue B, Nelson B, Thompson A, et al. The potential impact of COVID-19 on psychosis: a rapid review of contemporary epidemic and pandemic research. Schizophr Res. 2020;222:79-87.

68. McGrath J, Saha S, Welham J, El Saadi O, MacCauley C, Chant D. A systematic review of the incidence of schizophrenia: the 
distribution of rates and the influence of sex, urbanicity, migrant status and methodology. BMC Med. 2004;2:13.

69. Ferrando SJ, Klepacz L, Lynch S, Tavakkoli M, Dornbush R, Baharani R, et al. COVID-19 psychosis: a potential new neuropsychiatric condition triggered by novel coronavirus infection and the inflammatory response? Psychosomatics. 2020;61:551-5.

70. Rentero D, Juanes A, Losada CP, Álvarez S, Parra A, Santana V, et al. New-onset psychosis in COVID-19 pandemic: a case series in Madrid. Psychiatry Res. 2020;290:113097.

71. Parra A, Juanes A, Losada CP, Álvarez-Sesmero S, Santana VD, Martí I, et al. Psychotic symptoms in COVID-19 patients. A retrospective descriptive study. Psychiatry Res. 2020;291:113254.

72. Tariku M, Hajure M. Available evidence and ongoing hypothesis on corona virus (COVID-19) and psychosis: is corona virus and psychosis related? A narrative review. Psychol Res Behav Manag. 2020;13:701-4.

73. Mascolo A, Berrino PM, Gareri P, Castagna A, Capuano A, Manzo $\mathrm{C}$, et al. Neuropsychiatric clinical manifestations in elderly patients treated with hydroxychloroquine: a review article. Inflammopharmacology. 2018;26:1141-9.

74. Wada K, Yamada N, Sato T, Suzuki H, Miki M, Lee Y, et al. Corticosteroid-induced psychotic and mood disorders diagnosis defined by DSM-IV and clinical pictures. Psychosomatics. 2001;42: 461-6.

75. Mak IWC, Chu CM, Pan PC, Yiu MGC, Chan VL. Long-term psychiatric morbidities among SARS survivors. Gen Hosp Psychiatry. 2009;31:318-26.

76. Paterson RW, Brown RL, Benjamin L, Nortley R, Wiethoff S, Bharucha $\mathrm{T}$, et al. The emerging spectrum of COVID-19 neurology: clinical, radiological and laboratory findings. Brain. 2020;143: 3104-20.

77. Xie Q, Fan F, Fan X-P, Wang X-J, Chen M-J, Zhong B-L, et al. COVID-19 patients managed in psychiatric inpatient settings due to first-episode mental disorders in Wuhan, China: clinical characteristics, treatments, outcomes, and our experiences. Transl Psychiatry. 2020;10:1-11.

78. Wang D, Hu B, Hu C, Zhu F, Liu X, Zhang J, et al. Clinical characteristics of 138 hospitalized patients with 2019 novel coronavirus-infected pneumonia in Wuhan. China Jama American Medical Association. 2020;323:1061-9.

79. Li Y, Wang M, Zhou Y, Chang J, Xian Y, Mao L, et al. Acute cerebrovascular disease following COVID-19: a single center, retrospective, observational study. Stroke Vasc Neurol. 2020;5:279-84.

80. Correll CU, Detraux J, De Lepeleire J, De Hert M. Effects of antipsychotics, antidepressants and mood stabilizers on risk for physical diseases in people with schizophrenia, depression and bipolar disorder. World psychiatry. 2015;14:119-36.

81. Firth J, Cotter J, Torous J, Bucci S, Firth JA, Yung AR. Mobile phone ownership and endorsement of "mhealth" among people with psychosis: a meta- analysis of cross-sectional studies. Schizophr Bull. 2015;42:448-55.

82. Kasckow J, Felmet K, Appelt C, Thompson R, Rotondi A, Haas G. Telepsychiatry in the assessment and treatment of schizophrenia. Clin Schizophr Relat Psychoses. 2014;8:21-27A.
83. Zaami S, Marinelli E, Varì MR. New trends of substance abuse during COVID-19 pandemic: an international perspective. Front Psychiatry. 2020;11:700.

84. Becker WC, Fiellin DA. When epidemics collide: coronavirus disease 2019 (COVID-19) and the opioid crisis. Ann Intern Med. 2020;173:59-60.

85. Khatri UG, Perrone J. Opioid use disorder and COVID-19: crashing of the crises. J Addict Med. 2020;14:e6-7.

86. McCann Pineo M, Schwartz RM. Commentary on the coronavirus pandemic: anticipating a fourth wave in the opioid epidemic. Psychol Trauma Theory Res Pract Policy. 2020;12:S108-10.

87. Banducci AN, Weiss NH. Caring for patients with posttraumatic stress and substance use disorders during the COVID-19 pandemic. Psychol Trauma Theory Res Pract Policy. 2020;12:S113-4.

88. Ornell F, Moura HF, Scherer JN, Pechansky F, Kessler FHP, von Diemen L. The COVID-19 pandemic and its impact on substance use: implications for prevention and treatment. Psychiatry Res. 2020;289:113096.

89.• Wang QQ, Kaelber DC, Xu R, Volkow ND. COVID-19 risk and outcomes in patients with substance use disorders: analyses from electronic health records in the United States. Mol Psychiatry. 2020;14:1-10 A case-control study of electronic health records (EHRs) of over 73 million American patients identified 12,030 with a diagnosis of COVID-19 and compared COVID-19 patients with a co-morbid diagnosis of substance use disorder (SUD) to those without SUDs. COVID-19 patients with SUDs had significantly worse outcomes than COVID-19 patients without SUDs. In particular, African Americans and those with opioid use disorder (OUD) had increased risk for COVID-19 and suffering adverse outcomes, highlighting health disparities and the need to screen and treat SUDs.

90. Hulin J, Brodie A, Stevens J, Mitchell C. Prevalence of respiratory conditions among people who use illicit opioids: a systematic review. Addiction. 2020;115:832-49.

91. Tomashefski JF, Felo JA. The pulmonary pathology of illicit drug and substance abuse. Curr Diagn Pathol. 2004;10:413-26.

92. Schimmel J, Manini AF. Opioid use disorder and COVID-19: biological plausibility for worsened outcomes. Subst Use Misuse. 2020;55:1-2. https://doi.org/10.1080/10826084.2020.1791184.

93. Schuckit MA. Treatment of opioid-use disorders. N Engl J Med United States. 2016;375:1596-7.

94. Donroe JH, Bhatraju EP, Tsui JI, Edelman EJ. Identification and management of opioid use disorder in primary care: an update. Curr Psychiatry Rep. Springer; 2020;22:23. This is an important update for Primary Care providers that summarizes current literature and best practices in screening, diagnosis, and management of patients with opioid use disorder.

95. Mount Sinai Center for Post-COVID Care. Mount Sinai announces first-of-its-kind center for post-COVID care [Internet]. 2020. Available from: https://www.mountsinai.org/about/newsroom/ 2020/mount-sinai-announces-first-of-its-kind-center-for-postcovid-care-pr

Publisher's Note Springer Nature remains neutral with regard to jurisdictional claims in published maps and institutional affiliations. 